Erich J. Schwarz, Rainer Harms (Hrsg.)

Integriertes Ideenmanagement 
WIRTSCHAFTSWISSENSCHAFT 
Erich J. Schwarz, Rainer Harms (Hrsg.)

\section{Integriertes Ideenmanagement}

Betriebliche und überbetriebliche Aspekte unter besonderer Berücksichtigung kleiner und junger Unternehmen

Deutscher Universitäts-Verlag 
Bibliografische Information Der Deutschen Bibliothek

Die Deutsche Bibliothek verzeichnet diese Publikation in der Deutschen Nationalbibliografie; detaillierte bibliografische Daten sind im Internet über <http://dnb.ddb.de> abrufbar.

1. Auflage Oktober 2005

Alle Rechte vorbehalten

(C) Deutscher Universitäts-Verlag/GWV Fachverlage GmbH, Wiesbaden 2005

Lektorat: Ute Wrasmann / Anita Wilke

Der Deutsche Universitäts-Verlag ist ein Unternehmen von Springer Science+Business Media. www.duv.de

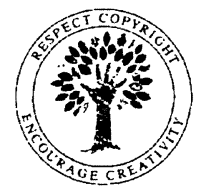

Das Werk einschließlich aller seiner Teile ist urheberrechtlich geschützt. Jede Verwertung außerhalb der engen Grenzen des Urheberrechtsgesetzes ist ohne Zustimmung des Verlags unzulässig und strafbar. Das gilt insbesondere für Vervielfältigungen, Übersetzungen, Mikroverfilmungen und die Einspeicherung und Verarbeitung in elektronischen Systemen.

Die Wiedergabe von Gebrauchsnamen, Handelsnamen, Warenbezeichnungen usw. in diesem Werk berechtigt auch ohne besondere Kennzeichnung nicht zu der Annahme, dass solche Namen im Sinne der Warenzeichen- und Markenschutz-Gesetzgebung als frei zu betrachten wären und daher von jedermann benutzt werden dürften.

Umschlaggestaltung: Regine Zimmer, Dipl.-Designerin, Frankfurt/Main

Gedruckt auf säurefreiem und chlorfrei gebleichtem Papier

ISBN-13:978-3-8350-0140-4

e-ISBN-13:978-3-322-82153-9

DOI: $10.1007 / 978-3-322-82153-9$ 


\section{Vorwort}

„Integriertes Ideenmanagement - Betriebliches und überbetriebliches Ideenmanagement unter besonderer Berücksichtigung kleiner Unternehmen" - unter dieser Überschrift steht die dritte Konferenz der Reihe „Entrepreneurship in Theorie und Praxis“ der Universität Klagenfurt. Wie auch die ersten beiden Konferenzen dieser Veranstaltungsreihe, im ersten und zweiten Halbjahr 2003, wird auch die diesjährige Konferenz von der Abteilung Innovationsmanagement und Unternehmensgründung in Zusammenarbeit mit der build! Gründerzentrum Kärnten $\mathrm{GmbH}$ veranstaltet.

Die Wirtschaft im deutschsprachigen Raum ist vor allem durch kleine und mittelgroße Unternehmen (künftig: KMU) gekennzeichnet. Beschäftigung und Wertschöpfung als Beispiele für positive gesamtwirtschaftliche Effekte werden zu einem großen Teil von KMU getragen. Damit auch in Zukunft der KMUSektor seine tragende Rolle erfüllen kann, ist es zwingend notwendig, dass sich diese Unternehmen durch Produkt- und Prozessinnovationen im Markt behaupten.

Allerdings zeigen empirische Befunde, dass kleine Unternehmen und Gründungsunternehmen großteils wenig innovativ sind. Die Ursachen hierfür sind vielfältig. So werden kleinen Unternehmen vielfach eine geringere technische Professionalität und unzureichende Kenntnisse von modernen Managementmethoden nachgesagt. In Bezug auf das Innovationsmanagement resultiert dies in einer mangelnden Fähigkeit eigene innovative Ideen zu entwickeln sowie Ideen aus dem Unternehmensumfeld zu beziehen. Aber auch mittlere und große Unternehmen berichten über Probleme im Management von Ideen. Hier sind es vor allem Schwierigkeiten bei der Bewertung und Auswahl von Ideen sowie bei der Verwertung von Ideen und Erfindungen, die nicht zur strategischen Ausrichtung des Unternehmens passen.

Wie die Instrumente des Ideenmanagements in KMU tatsächlich eingesetzt werden und wie mit den Schwierigkeiten des Ideenmanagements im Kontext dieser Unternehmen umgegangen werden kann, wird durch die Beiträge in diesem Tagungsband beleuchtet. In Bezug auf die im vorigen Abschnitt diskutierten Problemkreise liegt der Fokus der Beiträge dieser Tagung zum einem auf der internen Ideengenerierung und wirtschaftlichen Verwertung. Zum Zweiten wird der Transfer von Ideen und technologischem Wissen zwischen Unternehmen und ihrer Umwelt, wie kooperierenden Firmen und Forschungsinstitutionen, behandelt. Dabei werden ausführlich die Rollen der unterschiedlichen Akteure sowie deren Aktivitäten in regionalen Ideen- und Innovationssystemen diskutiert. 
Das Buch gliedert sich in vier Abschnitte. Ausgehend von Überlegungen zur Gestaltung des Wissenstransfers in technologieorientierten Kooperationen zwischen Universitäten und Unternehmen werden im ersten Teil die Aufgaben und Beiträge von Inkubatoren, Hochschulen und Technologieparks für die Gründung und Entwicklung innovativer KMU skizziert.

Die beiden folgenden Abschnitte widmen sich dem Management von Ideen und Erfindungen. Während im zweiten Teil der Fokus auf Unternehmensnetzwerke und große Unternehmen gelegt wird, behandeln die Beiträge des dritten Abschnitts das Ideenmanagement in neu gegründeten und kleinen Unternehmen.

Die Umsetzung von Ideen und Erfindungen in Produkte und Verfahren sowie deren Einführung in Märkte bzw. in Unternehmen ist Aufgabe des betrieblichen Innovationsmanagements. Im letzten Abschnitt dieses Buches werden ausgewählte Innovationskonzepte und -instrumente diskutiert.

Abschließend möchten wir allen Autorinnen und Autoren unseren herzlichen Dank aussprechen. Für die finanzielle Unterstützung bei der Drucklegung bedanken wir uns beim Mitveranstalter der Konferenz, der build! Gründerzentrum Kärnten $\mathrm{GmbH}$ und seinem Geschäftsführer, Herrn Dipl.-Ing. Siegfried Spanz, dem Amt der Kärntner Landesregierung, dem Förderverein des Instituts für Wirtschaftswissenschaften sowie den Projektpartnern des Projektes Innovin.

Besondere Anerkennung bei der Umsetzung dieses Buchprojektes gebührt unseren Kolleginnen Frau Christiane Krainer sowie Frau Mag. Gertraud Hellwagner-Beham.

Erich J. Schwarz und Rainer Harms 


\section{Inhaltsverzeichnis}

Erich J. Schwarz, Rainer Harms

Vorwort

V

Teil I: Inkubatoren und Wissenstransfer ........................................................... 1

Alicia Ma. Bolivar-Cruz, Rosa Mat. Batista-Canino,

Desiderio J. Garcia-Almeida, Esther Hormiga-Pérez

Knowledge transfer in technological cooperation agreements in the

context of SME's: Cooperative research $v s \mathrm{R} \& \mathrm{D}$ contracts.

Joanne L. Scillitoe, Alok K. Chakrabarti

Accelerated technological learning of new technology-based

ventures: The impact of social ties with technology incubator management

Benjamin B. Gansel, Matthias G. Raith, Helge M. Wilker

Die Hochschule als regionaler Impulsgeber für Unternehmensgründungen:

Eine empirische Untersuchung des Technologietransfers der Universität

Magdeburg von 1990 bis 2004

Giada Palamara

Science parks as innovative development catalysers for SMEs

Teil II: Ideenmanagement in großen Unternehmen und Netzwerken

Albert Vollmer, Theo Wehner, Wolfgang G. Weber

Sonet - Sozialorientiertes Netzwerk für Ideenaustausch: Konzept und

Erfahrungsbericht.

Roland A. Waldner

Verwertung von Ideen und Erfindungen am Beispiel PHILIPS DAP

Klagenfurt

Reinhard Schulte, Silke Tegtmeier, Fabian Eggers, Mareike Deutschmann

Von der Innovation zum Corporate Spin Off - Technologieorientierte Ausgründungen aus Großunternehmen in Deutschland. 
Teil III: Ideenmanagement in KMU

Marion A. Weissenberger-Eibl, Stephan Speith

Der tatsächliche Wert von Ideen in kleinen und neu gegründeten

Unternehmen

Kai-Ingo Voigt, Alexander Brem

Integriertes Ideenmanagement als strategischer Erfolgsfaktor

junger Technologieunternehmen

Martin Kaschny, Timo Podgorski

Innerbetriebliches Vorschlagswesen und Marketing: Eine Best-Case-Study ... 201

Gerald Steiner

Organizational Creativity Management.

Teil IV: Innovationskonzepte und -instrumente.

Erich Zahn, Patrice Lienhard

Strategisches Unternehmertum

Katrin Talke, Sören Salomo

Management von Initiativen für Innovationen in KMUs

Alfred Spielkamp, Christine Volkmann

Führungsinstrumente im Innovationsmanagement kleiner und

mittlerer Unternehmen

Jörn-Axel Meyer

Bekanntheit und Einsatz von Innovationsmethoden in jungen KMU:

Ergebnisse einer regelmäßigen Befragung im Zeitvergleich

Verzeichnis der Autoren 